\title{
Changes in Sef Levels Influence Auditory Brainstem Development and Function
}

\author{
Victoria E. Abraira, ${ }^{1 \star}$ Naomi Hyun, ${ }^{1 \star}$ Andrew F. Tucker, ${ }^{1 \star}$ Donald E. Coling, ${ }^{3}$ M. Christian Brown, ${ }^{4}$ Cindy Lu, ${ }^{1}$ \\ Gregory R. Hoffman, ${ }^{2}$ and Lisa V. Goodrich ${ }^{1}$ \\ Departments of ${ }^{1}$ Neurobiology and ${ }^{2}$ Cell Biology, Harvard Medical School, Boston, Massachusetts $02115,{ }^{3}$ Center for Hearing and Deafness, State University \\ of New York at Buffalo, Buffalo, New York 14260, and ${ }^{4}$ Eaton-Peabody Laboratory, Massachusetts Eye and Ear Infirmary, Boston, Massachusetts 02115
}

During development of the CNS, secreted morphogens of the fibroblast growth factor (FGF) family have multiple effects on cell division, migration, and survival depending on where, when, and how much FGF signal is received. The consequences of misregulating the FGF pathway were studied in a mouse with decreased levels of the FGF antagonist Sef. To uncover effects in the nervous system, we focused on the auditory system, which is accessible to physiological analysis. We found that the mitogen-activated protein kinase pathway is active in the rhombic lip, a germinal zone that generates diverse types of neurons, including the cochlear nucleus complex of the auditory system. Sefis expressed immediately adjacent to the rhombic lip, overlapping with FGF15 and FGFR1, which is also present in the lip itself. This pattern suggests that Sef may normally function in non-rhombic lip cells and prevent them from responding to FGF ligand in the vicinity. Consistent with this idea, overexpression of Sef in chicks decreased the size of the auditory nuclei. Cochlear nucleus defects were also apparent in mice with reduced levels of Sef, with $13 \%$ exhibiting grossly dysmorphic cochlear nuclei and $26 \%$ showing decreased amounts of GFAP in the cochlear nucleus. Additional evidence for cochlear nucleus defects was obtained by electrophysiological analysis of Sef mutant mice, which have normal auditory thresholds but abnormal auditory brainstem responses. These results show both increases and decreases in Sef levels affect the assembly and function of the auditory brainstem.

Key words: FGF; Sef; rhombic lip; cochlear nucleus; auditory brainstem response; central auditory processing defect

\section{Introduction}

During neural development, fibroblast growth factors (FGFs) exert effects on gene expression, proliferation, and survival (Dono, 2003). Distinct biological effects correlate with the degree of mitogen-activated protein (MAP) kinase pathway activation (Sato and Nakamura, 2004). MAP kinase levels are controlled by multiple feedback-induced antagonists, including Sprouties (Spry) and Sef (Tsang and Dawid, 2004). Sef is induced by FGF signaling and antagonizes the MAP kinase pathway cellautonomously (Furthauer et al., 2002; Tsang et al., 2002; Tsang and Dawid, 2004), either by preventing phosphorylation of the FGF receptor or by sequestering activated Erk1/2 in the cytoplasm (Kovalenko et al., 2003; Yang et al., 2003; Preger et al., 2004; Torii et al., 2004). Sef overexpression blocks activation of

\footnotetext{
Received Aug. 11, 2006; revised March 1, 2007; accepted March 5, 2007.

This work was supported by National Institutes of Health Grant R01 MH60612 (M. Tessier-Lavigne and W. C. Skarnes), National Institute on Deafness and Other Communication Disorders Grant 01089 (M.C.B.), The Medical Foundation Grant MED111090302 (L.V.G.), Alfred P. Sloan Foundation Grant BR-4261 (L.V.G.), and by the Mathers Charitable Foundation (L.V.G.). We are grateful to Donna Fekete for valuable input to every aspect of the project; to Kent Morest for introducing us to the anatomy of the cochlear nucleus; to Richard Wingate for providing the chick CMafb probe; to Siew-Lan Ang, Susan Dymecki, and Anna Farago for stimulating discussions and for sharing preliminary results; to John Blenis; and to Rosalind Segal for advice and comments on this manuscript. This work was begun with the helpful guidance and support of Marc Tessier-Lavigne and William Skarnes.

*V.E.A., N.H., and A.F.T. contributed equally to this work.

Correspondence should be addressed to Dr. Lisa V. Goodrich at the above address. E-mail: Lisa_Goodrich@hms.harvard.edu.

DOI:10.1523/JNEUROSCI.3477-06.2007

Copyright $\odot 2007$ Society for Neuroscience $\quad 0270-6474 / 07 / 274273-10 \$ 15.00 / 0$
}

the MAP kinase pathway (Kovalenko et al., 2003; Preger et al., 2004; Torii et al., 2004). Conversely, loss of Sef in morphant zebrafish or by RNA interference knockdown in cultured cells causes phenotypes consistent with excess FGF signaling (Furthauer et al., 2002; Tsang et al., 2002; Torii et al., 2004).

Mutations in FGF antagonists cause subtle phenotypes, most likely because of the presence of multiple, redundant regulators. Spry1 and Spry2 mutant mice exhibit normal body patterning with a few discrete phenotypes (Basson et al., 2005; Shim et al., 2005; Taketomi et al., 2005). Similarly, Sef mutant mice are viable with patterning defects apparent only when Spry is also inhibited (Lin et al., 2005). CNS phenotypes have not been described in Spry or Sef mutant mice, despite the clear role for FGF signaling here.

The auditory system offers an excellent opportunity to identify subtle neural defects, because it is amenable to physiological analysis. Sound is detected by hair cells in the cochlea. Spiral ganglion neurons carry sound information from the ear to cochlear nucleus neurons in the auditory brainstem. The cochlear nuclei are obligatory synaptic stations in the auditory pathway, receiving all sound input from a single ear, performing initial transformations of the stimulus, and routing this information bilaterally for further processing. A major source of cochlear nucleus neurons is the rhombic lip, a germinal zone that contains a diverse population of progenitors for several brainstem nuclei (Rodriguez and Dymecki, 2000; Wingate, 2001; Landsberg et al., 2005; Machold and Fishell, 2005; Wang et al., 2005; Farago et al., 
2006). The molecular heterogeneity of the rhombic lip suggests that secreted signals may regulate cell fate decisions here (Timmer et al., 2002; Landsberg et al., 2005). Indeed, FGF2 induces proliferation and migration of cochlear nucleus precursors from the rhombic lip in vitro (Zhou et al., 1996; Hossain et al., 2006). Thus, changes in FGF signaling could affect rhombic lip development and may result in changes in sound perception.

Here, we report that components of the FGF pathway are present in the rhombic lip, with Sef in the adjacent cell population. Gain-of-function and loss-of-function manipulations cause anatomical and physiological defects in the auditory brainstem. These results suggest that regulation of the FGF pathway by Sef contributes to the assembly and function of the auditory brainstem.

\section{Materials and Methods}

Mice. The KST223 gene trap line was reported previously (Leighton et al., 2001). The mice had been maintained for more than five generations on the C57BL6/J background. Genotyping was performed using the following primers: Int3-F2 (5'-GCCAAGCCTTGATATGACAAAC-3'), Int3-R1 (5'-TTATGAGTCATTCTCCAGCCCG-3'), and GTR1 (5' GGTCTTTGAGCACCAGAGGACATC-3'). Int3F2 and Int3R1 amplify a 594 bp band from wild-type DNA; Int3F2 and GTR1 amplify a 485 bp band specific to the KST223 insertion. To obtain timed pregnancies, matings were set up in the afternoon, and plugs were checked the next morning. The day of plug was called embryonic day 0.5 (E0.5). The mice were maintained in accordance with institutional and National Institutes of Health (NIH) guidelines approved by the Institutional Animal Care and Use Committee at Harvard Medical School.

Quantitative PCR. cDNA was made from total RNA using random primers. Long and/or short forms of Sef were amplified with SYBR Green Supermix (Bio-Rad, Hercules, CA) and the following primers: LVG081 (5'-CCCTGGGCTACATTTACTGC-3'), LVG082 (5' -ACCGTCTCCCGTACACTGAC-3'), LVG099 (5'-AGTCATCCTGGAGGAGCTGA-3'), LVG100 (5' -ATGGAAGGGAAAGGGACAAT-3'), LVG101 (5' CGTGGCTTTCCTATCCAAGA-3'), and LVG102 (5' -CACCCCACCACCTACAAAAG- $\left.3^{\prime}\right)$. Levels were normalized based on amplification of glyceraldehyde-3-phosphate dehydrogenase (GAPDH) from the same samples: GAPDHe-F (5'-CTCATGACCACAGTCCATGC-3') and GAPDHe-R (5'-GCACGTCAGATCCACGAC-3'). PCR was performed on a Chromo4 Continuous Fluorescence Detector and a PTC200 gradient cycler (MJ Research, Cambridge, MA) using a touchdown strategy, starting with a $10 \mathrm{~min}$ denaturation at $95^{\circ} \mathrm{C}$ and then decreasing from an annealing temperature of $70^{\circ} \mathrm{C}$ down to $60^{\circ} \mathrm{C}$ in $2^{\circ}$ intervals for five cycles beginning with $94^{\circ} \mathrm{C}(30 \mathrm{~s}), 70^{\circ} \mathrm{C}(30 \mathrm{~s})$, and $72^{\circ} \mathrm{C}(1 \mathrm{~min})$. The reaction proceeded for 30 cycles of $94^{\circ} \mathrm{C}(30 \mathrm{~s}), 60^{\circ} \mathrm{C}(30 \mathrm{~s})$, and $72^{\circ} \mathrm{C}(1 \mathrm{~min})$. Specificity was confirmed by examining the melting curves for each primer pair and by running products on an agarose gel. In all cases, single bands of the expected size and intensity were observed.

In situ hybridization. Nonradioactive in situ hybridization was performed on $14 \mu \mathrm{m}$ cryosections using the following probes: Math1 (Gray et al., 2004), Sef (Lin et al., 2002), Pax2, mouse Mafb (Gray et al., 2004), chick Mafb (Kataoka et al., 1994), barhl1, FGF15 (Wright et al., 2004), FGFR1 (Yamaguchi et al., 1992), FGFR2 (Peters et al., 1992), FGFR3 (Peters et al., 1993), and FGFR4 (nucleotides 2368-3323, GenBank accession number BC033313). A detailed protocol is available at http://goodrich.med.harvard.edu/index.htm.

$X$-gal staining. Staining for $\beta$-galactosidase activity was performed as described previously (Leighton et al., 2001), except that $15-20 \mu \mathrm{m}$ frozen sections were used and the tissue was fixed for $2 \mathrm{~h}$.

Immunohistochemistry. For phosphorylated Erk1/2 antibody stains, embryos were dissected in 0.01 m PBS (Invitrogen, San Diego, CA) and fixed in $4 \%$ paraformaldehyde in $0.01 \mathrm{M}$ PBS for $2 \mathrm{~h}$ at $4^{\circ} \mathrm{C}$. For other antibodies, embryos were fixed for $2 \mathrm{~h}\left(4^{\circ} \mathrm{C}\right)$ in $4 \%$ paraformaldehyde in $67 \mathrm{~mm}$ Sorenson's PBS. After overnight incubation in a primary antibody, Alexa-conjugated secondary antibodies (1:2000; Invitrogen) and a NeuroTrace counterstain (1:200; Invitrogen) were added for $2 \mathrm{~h}$ at room temperature or overnight at $4^{\circ} \mathrm{C}$. Tissue was imaged using a Nikon (Tokyo, Japan) E800 compound microscope or a Zeiss (Thornwood, NY) LSM510 confocal microscope. The following antibodies were used: anti-phosphorylated Erk1/2 (1:500; Biosource, Camarillo, CA), rabbit anti- $\beta$-galactosidase (1:2500; MP Biomedicals, Solon, $\mathrm{OH})$, goat anti- $\beta$-galactosidase (1:2500; Biogenesis, Sandown, NH), antiMafb (1:1500; Bethyl Laboratories, Montgomery, TX), anti-green fluorescent protein (GFP; 1:500, Invitrogen), and anti-GFAP (1:2000; Dako, Carpenteria, CA).

Chick electroporation. The Sef coding sequence (AF494209) was cloned into the SalI and BamHI sites of the pIRES2_eGFP vector (Invitrogen), downstream of a cytomegalovirus promoter. For controls, the empty pIRES2_eGFP vector was used. Because the enhanced GFP (eGFP) signal was found to be weak in the first 70 electroporated embryos, the subsequent 278 embryos were coelectroporated with plasmid JPA5, which encodes a yellow fluorescent protein (YFP)-CD8 protein that was brightly fluorescent in unfixed, living embryos. Electroporations were performed essentially as described previously (Krull, 2004). A concentration of $4 \mu \mathrm{g} / \mu \mathrm{l}$ DNA was injected into the lumen of the neural tube at the level of rhombomeres 5 and 6 in an E2 [Hamburger-Hamilton (HH) stage 11] chick embryo. A CUY-21 electroporator (Protech International, San Antonio, TX) was used to generate five $50 \mathrm{~ms}$ pulses of $10 \mathrm{~V}$, with $10 \mathrm{~ms}$ intervals between each shock. Electrodes were positioned so that DNA was taken up only on the right side of the neural tube. A total of 348 electroporations were performed (195 with Sef plasmid, 153 with empty vector). Two hundred seventy-eight embryos were scored for survival and YFP expression the following day: 193 survived (69\%), and of these, 154 expressed YFP (80\%). Forty-five embryos were collected for analysis on E4, and 45 embryos were collected for analysis on E13 (HH stage 39). Embryos were processed for immunohistochemistry with antiGFP antibodies (E4) as described above or for in situ hybridization with a probe to chick Mafb (E13).

Analysis of nucleus magnocellularis and nucleus angularis. The areas of nucleus magnocellularis (NM) and nucleus angularis (NA) were determined using Image 1.33 (NIH, Bethesda, MD) to outline the cMafbexpressing regions in $10 \times$ images of $14-\mu \mathrm{m}$-thick sections in the transverse plane. To avoid bias attributable to variations in section angle and the intensity of the $c M a f b$ signal, equivalent regions of the electroporated and unelectroporated sides were photographed and analyzed independently and were compared within a single embryo. For NA, areas were calculated on seven evenly spaced sections (approximately every $50 \mu \mathrm{m}$ ) in the central $80 \%$ of the nucleus. $c M a f b$ is expressed in a high rostral-low caudal gradient in NM, making it difficult to reliably calculate the area of the caudal tip of this nucleus. Therefore, NM areas were calculated on six evenly spaced sections in the rostral $75 \%$ of the nucleus. Areas were determined by two independent investigators blind to condition. An area ratio was calculated by dividing the sum of areas on the electroporated side over the sum of areas from the unelectroporated side. A length ratio was calculated by dividing the number of sections containing each nucleus on the electroporated side over the number on the unelectroporated side. The final ratio is the product of the area ratio and the length ratio and therefore reflects an estimate of the change in total volume on the electroporated side versus the unelectroporated side. Eleven control and seven Sef-electroporated embryos were excluded from the analysis because the in situ hybridization signal was too weak to reliably outline the nucleus or because too many sections were damaged to gain a reliable estimate of volume.

Histology. For Nissl staining, $14 \mu \mathrm{m}$ cryosections were dehydrated through an ethanol series, passed through xylenes, stained in $0.1 \%$ cresyl violet, destained in $1 \%$ glacial acetic acid, dehydrated in ethanol, and mounted with CytoSeal. To quantify the volume of the ventral cochlear nucleus $(\mathrm{VCN})$, the nucleus was divided into 11 equal bins from the rostral pole of the anterior VCN (AVCN) to the end of the posterior VCN (PVCN), which was recognized by the presence of the granule cell layer that separates it from the dorsal cochlear nucleus (DCN). ImageJ 1.33 (NIH) software was used to outline VCN in one section from each of the central nine bins; the largest value in each series was discarded because PVCN was frequently difficult to define reliably in sections where the 


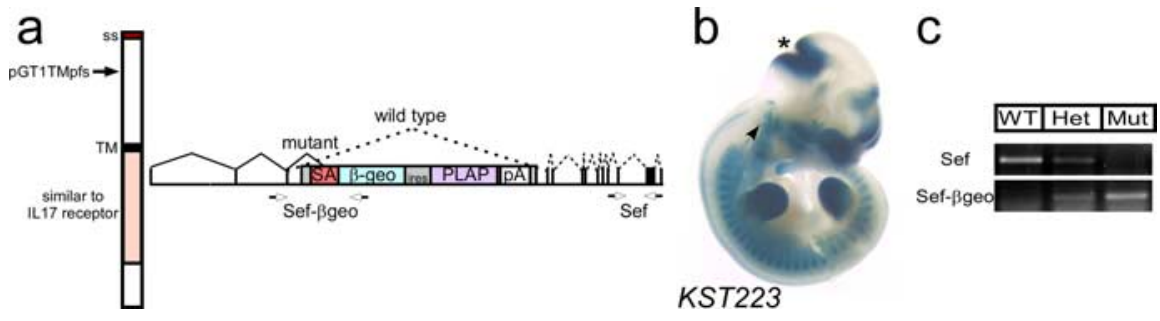

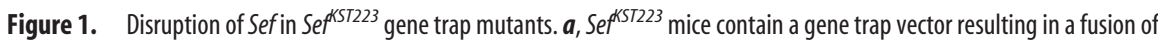
$\beta$-geo to Sef protein at the position indicated (Leighton et al., 2001; Mitchell et al., 2001). Primers specific for Sef- $\beta$-geo and for the $3^{\prime}$ end of Sef are indicated by arrows. SS, Signal sequence; TM, transmembrane domain; IL 17, interleukin 17; SA, splice acceptor. $\boldsymbol{b}, \mathrm{X}$-gal staining of an E10 Sef ${ }^{\alpha S T 223}$ heterozygous embry0. Sef- $\beta$-geo activity is seen in many regions of FGF signaling, including the mid-hindbrain junction (asterisk) and the ventral half of the otic vesicle (arrow). c, Reverse transcription-PCR of E14.5 RNA collected from wild-type (WT), heterozygous (Het), and mutant (Mut) embryos with primers specific for Sef shows a moderate decrease in Sef expression levels in heterozygous embryos and a strong decrease in mutants. Conversely, Sef- $\beta$-geo is present in heterozygotes and mutants but not wild types.

granule cell layer was sectioned tangentially. The eight remaining values were summed to provide an estimation of volume.

Measurements of hearing. Auditory brainstem responses (ABRs) in response to click stimuli (Shim et al., 2005) and Distortion Product Otoacoustic Emissions (DPOAE) and ABRs in response to pure tones (Liberman et al., 2002) were obtained as described previously. TuckerDavis Technologies (Alachua, FL) software was used to measure latencies and amplitudes from waveforms obtained in response to clicks. Responses to pure tone stimuli were analyzed by determining the lowest sound pressure level (SPL) at which each peak could be detected.

Statistics. Statistical significance was determined by single-factor ANOVA comparison or the one-tailed Mann-Whitney $U$ test of wildtype, heterozygote, and homozygote populations with an $\alpha$ of 0.05 . A result was considered significant if the $p$ value was $<0.05$.

\section{Results}

Generation of a Sef mouse mutant by gene trap mutagenesis We characterized a Sef mutant mouse line that was generated during the course of a large-scale gene trap mutagenesis screen (Leighton et al., 2001; Mitchell et al., 2001). The KST223 mutant mouse line contains an insertion of the GTITMpfs gene trap vector into the third intron of the Seflocus, resulting in a fusion of $\beta$-geo to Sef at nucleotide 332 (Fig. 1a). X-gal staining revealed Sef- $\beta$-geo fusion protein activity in sites of FGF signaling and was consistent with previous reports of Sef expression patterns (Fig. $1 b$ and data not shown). Reverse transcription-PCR of embryonic and adult brain cDNA shows that almost no wild-type Sef transcript is present in homozygotes, which instead express high levels of Sef-- $\beta$-geo (Fig. 1c). As determined by real-time PCR for exons shared by the two predicted splice forms, E14.5 Sef ${ }^{\text {KST223 }}$ homozygotes $(n=2)$ have $8.97 \pm 1.74 \%$ of wild-type transcript, and adult brain $(n=5)$ has $16.08 \pm 5.34 \%$ transcript remaining. In heterozygotes, Seflevels are mostly normal but are highly variable $[104.5 \pm 31.03 \%$ at E14.5 $(n=4) ; 74.36 \pm 31.07 \%$ in adults $(n=3)]$. The wide range of Sef levels observed in heterozygotes could reflect variable feedback-induced regulation of Sef, which is itself a target of the FGF pathway. Amplification with primers specific for each variant (Preger et al., 2004) confirms that both splice forms are equally affected at embryonic and adult stages (data not shown).

\section{The FGF pathway is active in the developing auditory brainstem}

Identification of defects in Sef mutant mice has been complicated by the presence of multiple inhibitors in the FGF pathway. Indeed, previous studies of Sef mutant mice showed that Sprouty antagonists compensate for Sef during early embryonic develop- ment (Lin et al., 2005). Similarly, the $S e f^{\text {XST223 }}$ gene trap mutants are viable and fertile with no gross developmental defects. To uncover any subtle phenotypes, we focused on a discrete region of the nervous system for which there is a clear functional readout: the cochlear nucleus complex, which is an obligatory synaptic station for all auditory input from the inner ear.

Most cochlear nucleus precursors originate in the rhombic lip, a germinal zone adjacent to the roof plate (Fig. 2a). To determine whether the FGF pathway might play a role in cochlear nucleus development, we examined the distribution of Sef and other components of the FGF pathway here. At E11.5, just when the first cochlear nucleus cells are born, little to no Sef expression is observed in the neural tube at the level of the inner ear (data not shown). Beginning at E12.5 and persisting through E15.5, Sef is expressed in a ventral region of the ventricular zone and in a dorsal domain that surrounds the roof of the fourth ventricle (Figs. $2 b-d, 3 a$ and data not shown). Comparison to the rhombic lip marker Math1 and the VCN marker Mafb shows that Sef is present in cells adjacent to the auditory region of the rhombic lip, abutting the developing cochlear nucleus (Fig. $2 d-f$ ). A small number of cochlear nucleus neurons derive from non-lip regions at this level of the brainstem (Farago et al., 2006). To see whether Sef-positive cells adjacent to the rhombic lip might eventually populate the cochlear nuclei, we took advantage of the perdurance of Sef- $\beta$ geo activity to follow their short-term fate. Stained cells appear to stream from the dorsal expression domain toward the developing cochlear nucleus (Fig. 2g-i). However, because this activity is weak and transient, it was not possible to follow the final fate of these cells in the adult cochlear nucleus.

Next, we compared the expression of Sef to other components of the FGF pathway at E14.5, a time when many cochlear nucleus cells are being generated within the rhombic lip (Pierce, 1967). FGF15 is present overlapping with Sef-expressing cells in the hindbrain (Fig. 3a,c). The receptor FGFR1 is expressed throughout the ventricular zone and in the rhombic lip (Fig. 3b). Moreover, cells with nuclear Erk1/2 staining are present in the rhombic lip (Fig. 3d). These cells do not express Sef, consistent with the idea that Sef inhibits activation of the pathway. These observations support the model that FGF15 activates the MAP kinase pathway in the rhombic lip, whereas Sef inhibits activation in neighboring cells.

\section{Overexpression of Sef inhibits development of auditory nuclei in chicks}

To test the contribution of FGF signaling to cochlear nucleus development, we used an overexpression strategy in chicks. We reasoned that transient blockade of FGF signaling by overexpression of Sef could reveal a normal requirement for regulated FGF signaling in the brainstem. In chickens, NM and NA serve similar functions as the murine cochlear nucleus complex (Ryugo and Parks, 2003), are also proposed to originate from the rhombic lip (Harkmark, 1954; Cramer et al., 2000), and express cMafb (Fig. $4 c-f)$. For instance, surgical removal of the rhombic lip from E4-E5 chicks results in small cochlear nuclei (Harkmark, 1954). Moreover, chicken Sef is $80 \%$ identical to mouse Sef, shares a 
similar expression pattern, and is regulated by FGF signaling like its mouse ortholog (Harduf et al., 2005).

Sef or empty vector control constructs were electroporated along with a YFP expression plasmid into the developing hindbrain at the level of the otic vesicle of E2 chick embryos, a time when cells are being fated to contribute to the auditory nuclei (Cramer et al., 2000, 2004, 2006) and just before a crucial period of rhombic lip development (Harkmark, 1954). Successful electroporations resulted in the expression of YFP in rhombomere 5, with expression extending to variable degrees rostrally and caudally (Fig. 4a). By E4, YFP-positive cells were present in the rhombic lip at the level of the otic vesicle in all embryos examined ( $n=3$ control vectors and 3 Sef electroporated) (Fig. 4b). Thus, a subset of cells in the rhombic lip of rhombomere 5 always received vector, with many embryos showing additional expression in rhombomeres 4 and/or 6 . Expression was limited to the electroporated side.

To assess effects on the auditory brainstem, embryos were collected on day 13 (HH stage 39), sectioned, and hybridized with a probe to $c M a f b$. The overall size and morphology of the brain was normal in all embryos. No gross defects in either NM or NA were observed in control embryos $(n=12)$, although mild fluctuations in the size of the nuclei were apparent. In contrast, in seven Sef-electroporated embryos (of 15 analyzed), one or both auditory nuclei were noticeably smaller both in area and length on the electroporated side (Fig. $4 c-f$ ). The apparent reduction in size was quantified by comparing the area of $c M a f b$-positive cells in sections through $\mathrm{NM}$ and NA on treated and untreated sides of the embryos and by counting the number of sections that contained each nucleus. In a subset of embryos, the area of NM was consistently smaller, and the nucleus was shorter on the electroporated side of Sef-treated embryos, particularly in rostral regions (Fig. $4 g, h$ ). Volume ratios for the electroporated versus unelectroporated sides of the embryo were calculated (see Materials and Methods). The control population showed no significant deviation from the expected value of 1 , with average ratios of $1.01 \pm 0.08$ for NM and $1.06 \pm 0.09$ for NA (Fig. $4 i)$. However, the volume ratios were decreased in the Sefelectroporated population, with values of $0.91 \pm 0.17$ for $\mathrm{NM}$ and $0.96 \pm 0.17$ for NA ( $p<0.08$, Mann-Whitney $U$ test $)$. The effect was partially penetrant, most likely reflecting differences in the level and location of Sef overexpression in different electroporated embryos. Affected embryos were defined as those that had volume ratios $<0.8$. By these criteria, no control embryos were c-g, $50 \mu \mathrm{m} ; \boldsymbol{i}, 10 \mu \mathrm{m}$.
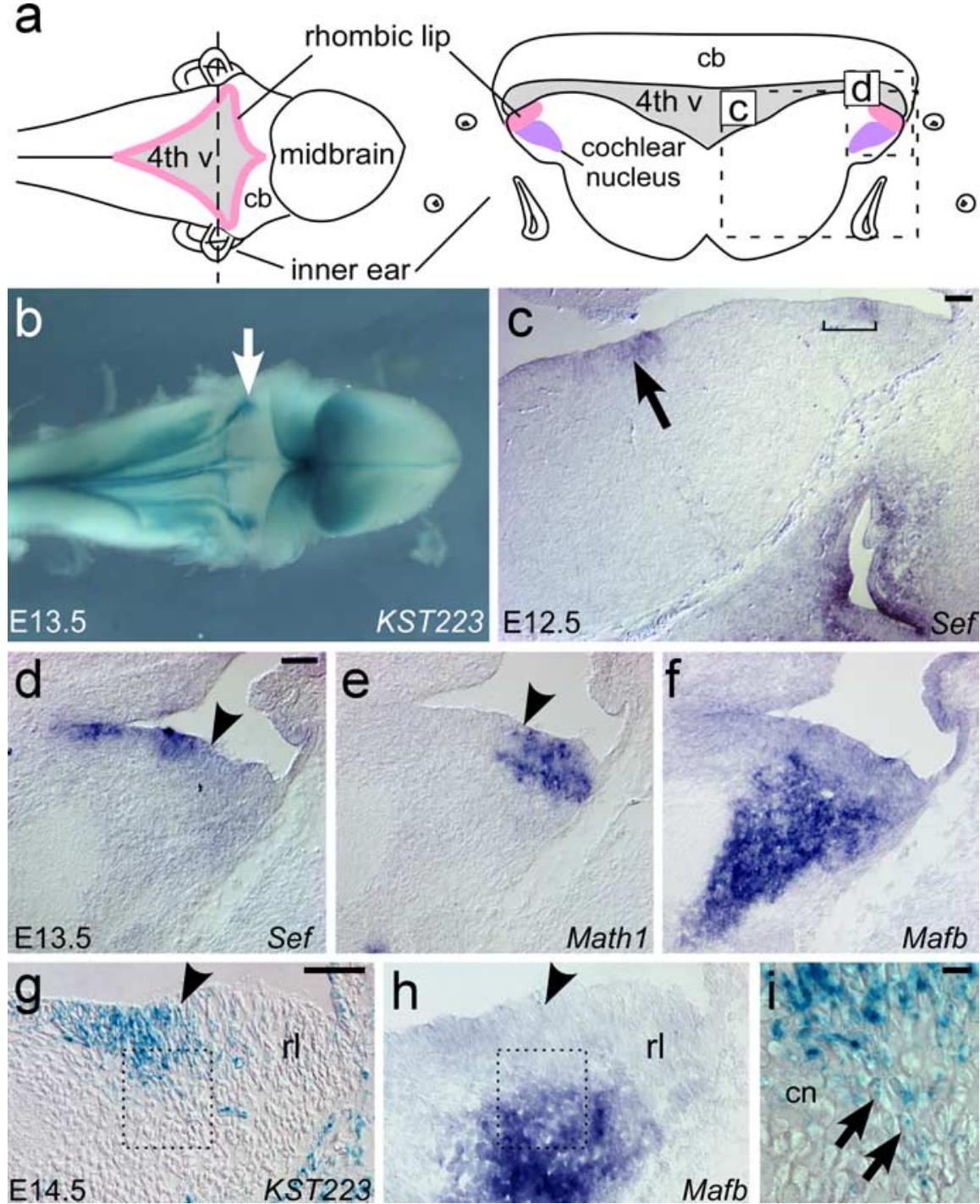

Figure 2. Sef is expressed in cells bordering the rhombic lip. $\boldsymbol{a}$, Dorsal (left) and transverse (right) diagrammatic views of the rhombic lip (pink) in an E12-E14 mouse embryo. The transverse view is through the plane indicated (dashed line); the position of the inner ear provides a guide for the rostrocaudal position in the hindbrain. At the level of the inner ear, the cochlear nucleus complex (purple) develops immediately adjacent to the rhombic lip. Dashed boxes outline the regions that are shown in $c$ and $\boldsymbol{d} \boldsymbol{- g}$. 4th v, Fourth ventricle; $c b$, cerebellum. $\boldsymbol{b}$, Dorsal view of an E13.5 Sef $\mathrm{fST223}^{\mathrm{k}}$ heterozygous embryo stained for $\beta$-galactosidase activity. Sef- $\beta$-geo is active in cells bordering the rim of the fourth ventricle, with intense activity (arrow) at the level of the inner ear, which has been dissected away. c, Transverse section of an E12.5 wild-type embryo hybridized with a probe to Sef. As indicated by X-gal staining, Sef is produced in a band of cells (bracket) adjacent to the rhombic lip and in a medial portion of the ventricular zone (arrow). $\boldsymbol{d}-\boldsymbol{f}$, Transverse adjacent sections through the E13.5 hindbrain hybridized with probes to Sef (d), Math1 (e), and $\operatorname{Mafb}(\boldsymbol{f})$. Sef is expressed adjacent to Math 1 in the rhombic lip (arrowheads mark the lateral limit of Math 1 expression) and abuts Mafb in the developing cochlear nucleus. $\boldsymbol{g}, \boldsymbol{h}$, Transverse semi-adjacent sections of an E14.5 Sef ${ }^{\text {kST223 }}$ heterozygous embryo stained for $\beta$-galactosidase activity $(\boldsymbol{g})$ and hybridized with a probe to $M a f b(\boldsymbol{h})$. As seen at earlier stages, $\beta$-galactosidase activity is detected in the Sef-positive region adjacent to the rhombic lip (rl) (arrowhead indicates boundary). In addition, weakly stained cells are present in the developing cochlear nucleus, as determined by the expression of Mafb on a semi-adjacent section $(\boldsymbol{h})$. The dashed boxes correspond to the region shown in $\boldsymbol{i}$. $\boldsymbol{i}$, High-power view of the region boxed in $\boldsymbol{g}$. A stream of stained cells with elongated morphology (arrows) appears to move from the Sef-positive region into the cochlear nucleus anlage (cn). Scale bars:

affected, but six Sef-electroporated embryos had smaller NM, with an average volume ratio of $0.72 \pm 0.1$, and two embryos had smaller NA, with an average ratio of $0.62 \pm 0.08$. One embryo had effects in both NM and NA, so overall, ectopic expression of Sef decreased the volume of the auditory nuclei in 7 of 15 embryos. The remaining embryos had an average ratio close to 1 (1.02 \pm 0.07 for NM, $1.03 \pm 0.04$ for NA). Consistent with this quantification, no cochlear nucleus defects were apparent in these em- 


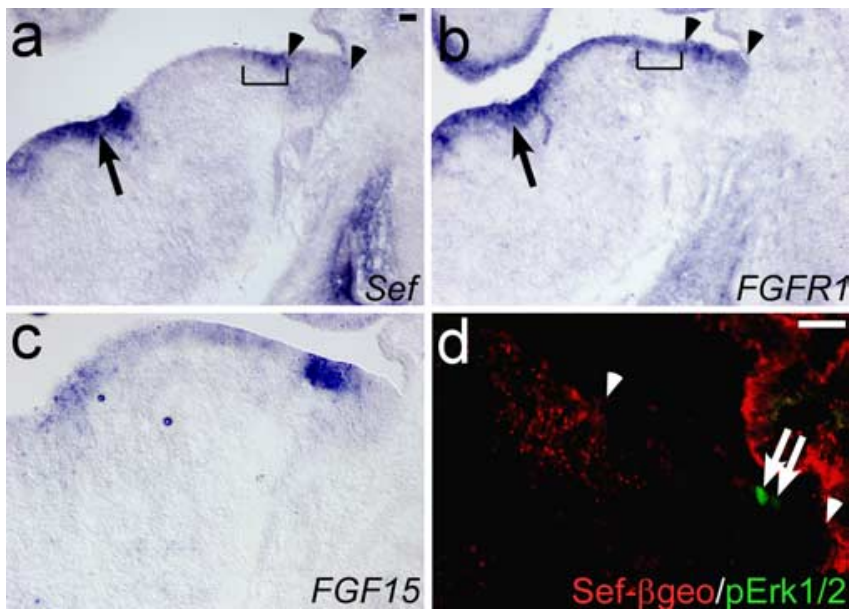

Figure 3. The FGF pathway is active in the rhombic lip. $\boldsymbol{a}-\boldsymbol{c}, \mathrm{E} 14.5$ wild-type tissue hybridized with probes to Sef $(\boldsymbol{a}), F G F R 1(\boldsymbol{b})$, and $F G F 15$ (c). $\boldsymbol{a}$ and $\boldsymbol{b}$ are adjacent sections. Sef (bracket) overlaps with FGFR1 near the rhombic lip (arrowheads) and in the medial floor of the fourth ventricle (arrows). FGF15 is expressed at high levels near the rhombic lip, overlapping Sef (ad-

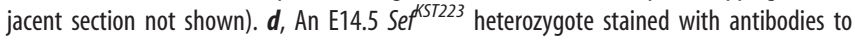
$\beta$-galactosidase (red) and phosphorylated Erk1/2 (pErk1/2; green). Phosphorylated Erk1/2positive cells (arrows) are present in the rhombic lip (arrowheads) but not in the Sef- $\beta$-geo expression domain, indicating that the MAP kinase pathway is active in the rhombic lip itself. All sections are transverse and correspond to the regions outlined in Figure 2. Scale bars, $50 \mu \mathrm{m}$.

bryos. The truncation of NM and NA in Sef-electroporated embryos is reminiscent of the effects of surgical removal of the rhombic lip (Harkmark, 1954) and suggests that FGF signaling affects the number of rhombic lip progenitors that assume auditory fates, much as has been proposed for Pax6 and the bone morphogenetic protein pathway in the development of precerebellar nuclei from more caudal regions of the rhombic lip (Landsberg et al., 2005).

\section{Low levels of Sef are associated with cochlear nucleus defects} Given the evidence that ectopic Sef can interfere with development of the auditory nuclei, we next asked whether the cochlear nucleus complex develops properly in $S e f^{K S T 223}$ mutant mice. We surveyed the overall organization of the cochlear nucleus in Nisslstained sections of the auditory brainstem from wild-type $(n=$ $5)$, heterozygous $(n=4)$, and homozygous $(n=11)$ adult animals. The cochlear nucleus complex has three major divisions: DCN, PVCN, and AVCN (Fig. 5a). We assessed four cell populations: granule cells, which are recognized by their small size and location in the microneuronal shell; octopus cells, which are clustered in a region of PVCN, have large cell bodies, and have dendrites oriented perpendicular to the entry of the eighth nerve; bushy cells, which are numerous in AVCN and express Mafb; and astrocytes, which produce GFAP.

Most mutants had histologically normal cochlear nuclei, with three distinct divisions and no obvious changes in the number or location of granule cells, octopus cells, or bushy cells (data not shown). However, one homozygote and one heterozygote had severe morphological defects (Fig. $5 b-d$ ). DCN was normal, but AVCN was disorganized and did not form a discrete nucleus. In addition, an outgrowth of the cerebellum covered its lateral surface. Although AVCN appeared larger in many sections, this most likely reflects the overall distortion of the mutant nucleus, because a systematic estimation of VCN areas throughout the rostrocaudal extent of the nucleus did not reveal any significant increase in size (wild type, $\left.6.65 \pm 1.14 \mathrm{~cm}^{2} ;-/-=6.89 \pm 1.11 \mathrm{~cm}^{2}\right)$. Based on the
Nissl-stained appearance of cells, cellular differentiation appeared to proceed properly. The nearby cerebellum was disorganized, but the superior olivary complex was normal (data not shown). To gain additional insight into this phenotype, we looked at the expression of math1, barhl1, and Mafb to see whether there were changes in the rhombic lip or its derivatives in E14.5 homozygotes. However, no changes were apparent in the dimensions of the rhombic lip (math1), the cochlear granule cell extramural stream (barhl1), or the anlage of VCN (Mafb) ( $n=2$ wild-type and 7 -/-; data not shown). The stages analyzed include the peak of cochlear nucleus cell production (E14.5) and its final mature structure (adulthood). However, we cannot rule out the presence of transient defects at other times that were not evaluated.

\section{Sef mutant mice respond poorly to high SPL stimuli}

An advantage of studying the auditory system is that hearing is easily tested in mice, making it possible to reveal defects in auditory function even in the absence of obvious anatomical defects. We therefore conducted a detailed analysis of ABRs, a commonly used measure of auditory system activity. The first ABR waveform peak (wave i) reflects the activity of spiral ganglion cells, whereas later peaks arise from the activity of neurons within the central auditory pathway. In the mouse, the correspondence between specific neuron groups and the later waves has not been completely established. However, work on the cat suggests that the generators of these later peaks are cells in the cochlear nucleus (wave ii), a combination of cochlear nucleus cells and superior olivary complex neurons (wave iii), medial superior olivary neurons (wave iv), and neurons in the inferior colliculus (wave v) (Zaaroor and Starr, 1991; Melcher and Kiang, 1996; Melcher et al., 1996a,b; Kaga et al., 1997).

Two ABR studies were performed to assess hearing in 12- to 14 -week-old Sef ${ }^{K S T 223}$ mice. First, ABRs were recorded in response to click stimuli ( $n=5$ wild type, $8+/-, 6-/-$ ) (Fig. $6 a$ ). Second, ABRs were recorded in response to pure tone stimuli ranging from 5 to $45 \mathrm{kHz}(n=4$ wild type, $8-/-)$ (Fig. $6 b)$. For click stimuli, wild types and homozygotes had similar thresholds $(14 \pm 10.33 \mathrm{~dB}$ in wild type vs $13.25 \pm 5.8 \mathrm{~dB}$ in $-/-$ ). Similarly, there was little difference in threshold at any frequency for wildtype and homozygous animals presented with pure tones (Fig. $6 c$ ). Outer hair cell function was also normal, with no shifts in DPOAE thresholds $[30 \pm 7.1 \mathrm{~dB}(n=3$ wild type $)$ vs $33.13 \pm 5.3$ $\mathrm{dB}(n=4-/-)]$ at $16 \mathrm{kHz}$.

Although thresholds were unchanged, mutant animals had qualitatively different responses to suprathreshold stimuli compared with wild types. Consistent with the presence of morphological defects in the cochlear nucleus, both homozygotes and heterozygotes were affected. Amplitudes and latencies of waves $i$ and ii were overlapping for all three genotypes, but the average distribution of responses for waves $\mathrm{i}$ and ii was significantly smaller in homozygote and heterozygote populations presented with stimuli louder than $55 \mathrm{~dB}$ ( $p<0.05$, ANOVA) (Fig. $6 d$ ). There was no change in latency for the first two peaks (for wave $\mathrm{i}$ : wild type, $1.52 \pm 0.08 \mathrm{~ms} ;-/+, 1.53 \pm 0.12 \mathrm{~ms} ;-/-, 1.56 \pm$ $0.17 \mathrm{~ms}$; for wave ii: wild type, $2.32 \pm 0.09 \mathrm{~ms} ;-/+, 2.30 \pm 0.17$ $\mathrm{ms} ;-/-, 2.34 \pm 0.11 \mathrm{~ms}$ ) (Fig. 6e). However, waves iii-v were frequently difficult to identify in response to either clicks or tone bursts, with significantly increased thresholds for wave iii in mutants (54.06 $\pm 15 \mathrm{~dB}$ ) compared with wild-type littermates $(38.13 \pm 10.7 ; p<0.02$, ANOVA) (Fig. $6 f)$. Waves iv and v were also significantly affected ( $p<0.02$, ANOVA). Of eight animals tested, four mutants had late wave thresholds $>50 \mathrm{~dB}$ in both ears and two had increased thresholds in one ear, suggesting a 

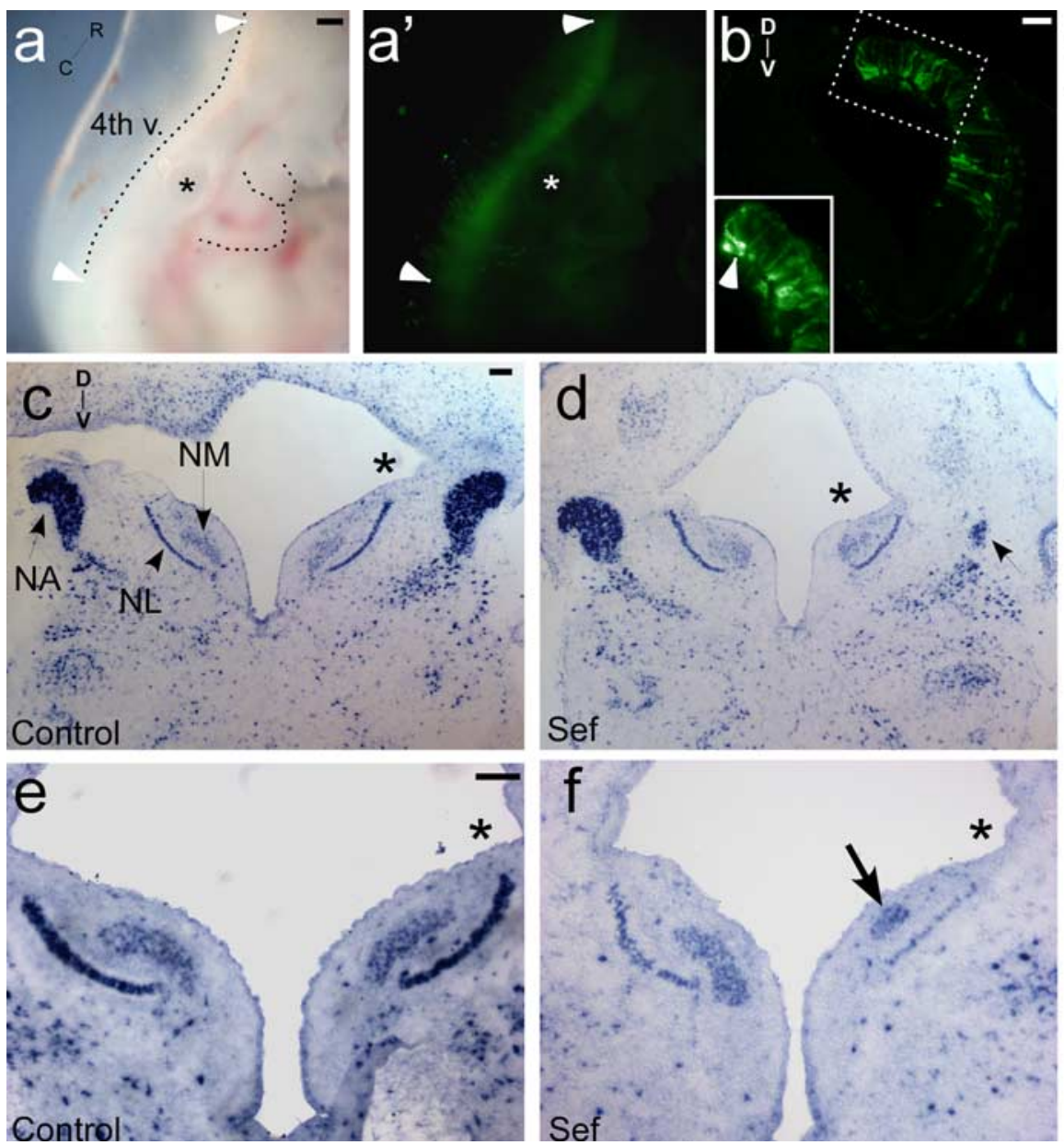

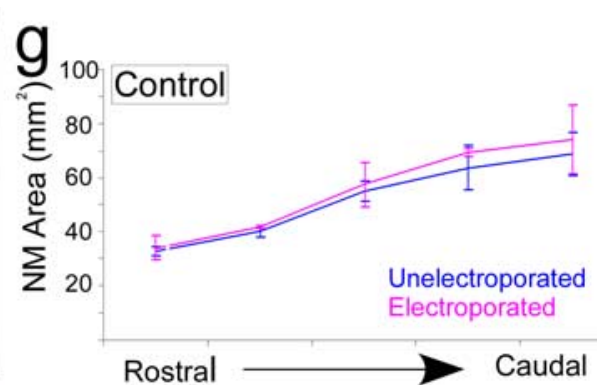

h

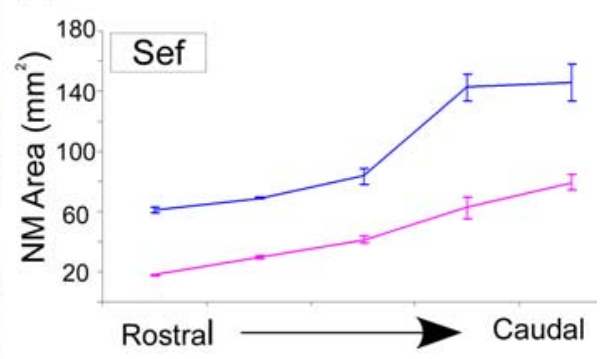

i

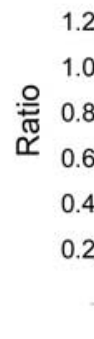

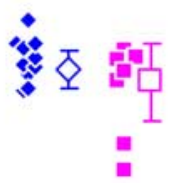

NM

NA

Figure 4. Overexpression of Sef inhibits auditory nucleus development in chicks. $\boldsymbol{a}, \boldsymbol{a}^{\prime}$, Bright-field $(\boldsymbol{a})$ and fluorescent $\left(\boldsymbol{a}^{\prime}\right)$ lateral views of an E4 electroporated chick embryo at the level of the otic vesicle (asterisk). Rostral (r) is up and to the right; caudal (c) is down and to the left. eGFP expression can be seen at the level of the otic vesicle and extending rostrally and caudally (arrowheads). 4th v, Fourth ventricle. $\boldsymbol{b}$, Transverse section through an eGFP-electroporated E4 chick embryo. Strongly labeled cells are present in the rhombic lip (arrowhead) and are also scattered throughout the neural tube, including commissural neurons that have extended axons toward the midline. Only the electroporated side is shown; no labeling is seen on the unelectroporated side. $\mathbf{c}-\mathbf{f}$, Coronal sections through control $(\boldsymbol{c}, \boldsymbol{e})$ and Sef-electroporated $(\boldsymbol{d}, \boldsymbol{f})$ embryos were hybridized with a probe to $\mathrm{cMafb}$. In control embryos, cMafb is produced in NM medially and in NA laterally. cMafb is also expressed in the nucleus laminaris, which serves a function equivalent to the medial superior olive in mammals. In NA-affected embryos ( $\boldsymbol{d}$ ), NA is drastically reduced (arrow) on the electroporated side (asterisk), but NM is unaffected. In NM-affected embryos ( $f$ ), NM is smaller on the electroporated side. Nucleus laminaris (NL) is variably affected, appearing to be shorter and slightly disorganized, but this phenotype was not robust enough to quantify. $\boldsymbol{g}, \boldsymbol{h}$, The area of NM (in square micrometers) was measured every $28 \mu \mathrm{m}$ on both sides of the brainstem, moving from rostral to caudal regions ( $x$-axis). Measurements are shown for the embryos depicted in $\boldsymbol{e}$ and $\boldsymbol{f}$. In the control embryo $(\boldsymbol{g})$, the area of NM is the same on the unelectroporated (blue) and electroporated (pink) sides. In the Sef-electroporated embryo ( $\boldsymbol{h}$ ), NM is smaller on the electroporated side in every section. $\boldsymbol{i}$, Individual (filled symbols) and average (open symbols) NM and NA ratios for control-electroporated (blue) and Sef-electroporated (pink) embryos. The volumes of NM and NA on the electroporated side were compared with the volumes on the unelectroporated side, with a value of 1 predicted as normal. Error bars indicate SD. Although no control-electroporated embryos had volume ratios $<0.8$, six Sef-electroporated embryos fell outside this range for NM, and two fell outside this range for NA. D, Dorsal; $V$, ventral.

penetrance of $\sim 50 \%$. In summary, Sef ${ }^{K S T 223}$ gene trap mice had normal auditory thresholds but did not generate appropriate responses in the brainstem, as reflected by the decreased amplitudes of waves i and ii and higher thresholds for later waves. Affected animals did not display any obvious changes in cochlear nucleus morphology or organization.

To see whether Sef activity in the mature cochlear nuclei may also contribute to the observed defects, we examined the expression of Sef at postnatal day 0 (P0), P6, P14, and adult stages. Beginning postnatally, Sef is present at high levels in the molecular layer and the microneuronal shell of the cochlear nucleus complex and at low levels in the magnocellular core (Fig. 7a). This pattern parallels the expression of GFAP (Fig. 7a). Indeed, Sef- $\beta$-geo-positive cells are non-neuronal and are GFAP positive (Fig. $7 b, c)$, so we conclude that Sef is produced by astrocytes in the cochlear nucleus. In other regions of the nervous system, FGF signaling from neurons is hypothesized to inhibit accumulation of GFAP in astrocytes. Consistent with this model, aged FGFR3 knock-out mice have abnormally high levels of GFAP in graymatter astrocytes (Pringle et al., 2003). We looked at the pattern of GFAP staining in animals that had been assessed by ABR recordings ( $n=4$ wild-type and $7-/-$ ). Remarkably, the two animals with the highest thresholds for wave iii $(55-80 \mathrm{~dB})$ had noticeably reduced staining relative to wild-type controls (Fig. $7 d-g$ ). GFAP staining was not significantly diminished in animals with more modest ABR effects. This result suggests that in addition to its functions during neuronal development, Sef may also contribute to proper regulation of FGF signaling in astrocytes in adult animals.

\section{Discussion}

Our results suggest that Sef regulation of FGF signaling influences both the development and function of the auditory brainstem. In 

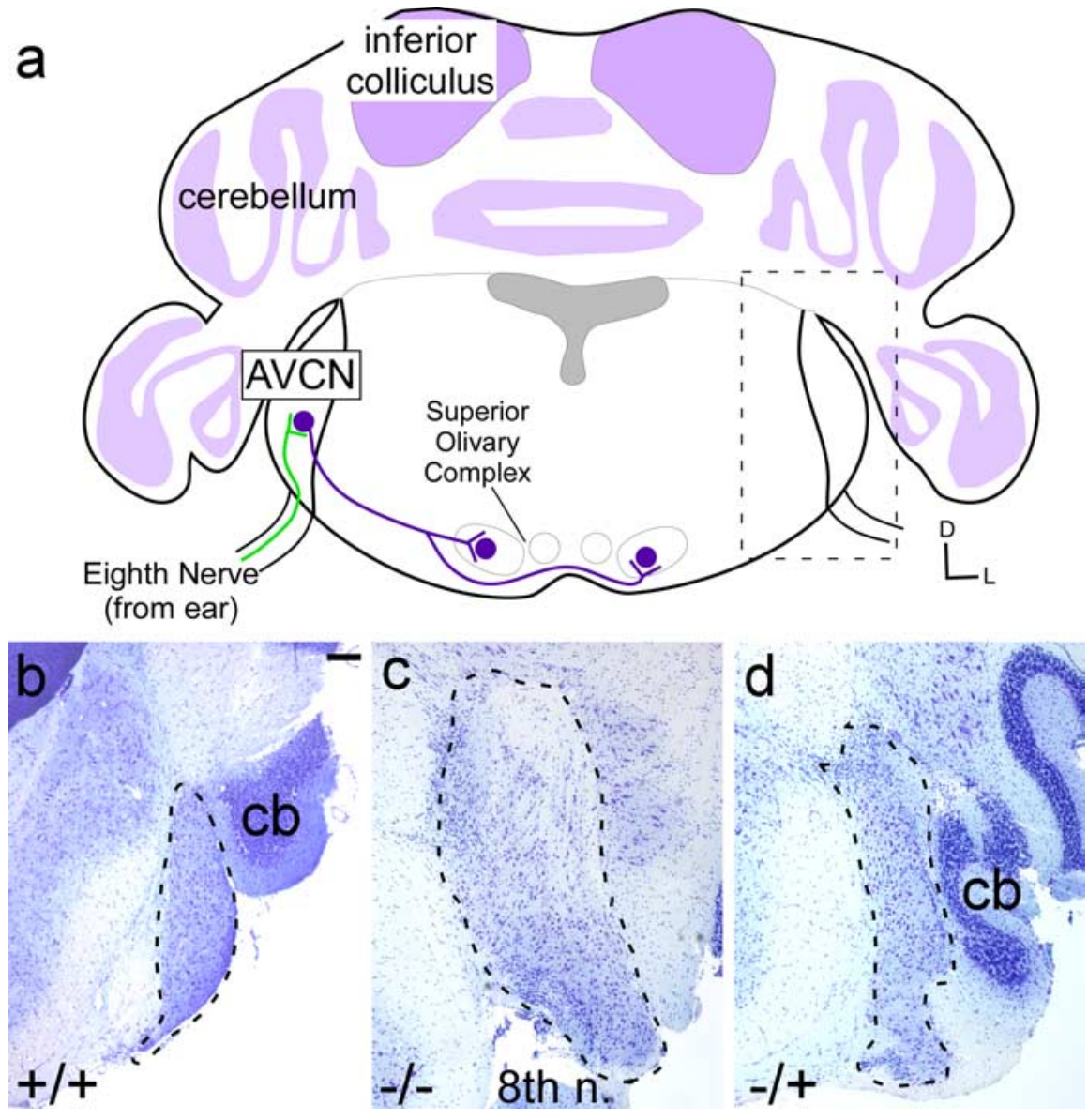

Figure 5. Cochlear nucleus defects in a subset of Sef mutant mice. $\boldsymbol{a}$, Diagram of the mouse auditory brainstem. Information arrives from the inner ear via the eighth nerve. Spiral ganglion axons (green) connect to a variety of cell types in both divisions of the cochlear nuclei. A major population of neurons in AVCN (purple) projects to the superior olivary complex both ipsilaterally and contralaterally; other pathways are not shown. The dashed box indicates the region shown in $\boldsymbol{b}-\boldsymbol{d}$. D, Dorsal; L, lateral. $\boldsymbol{b}-\boldsymbol{d}, \mathrm{A}$ Nissl-stained section in the coronal plane through a wild-type brainstem $(\boldsymbol{b})$ illustrates the normal anatomy of the cochlear nucleus. Abnormal morphology was observed bilaterally in one mutant (c) and unilaterally in one heterozygote (d). AVCN is misshapen and fails to separate from the surrounding tissue. The cerebellum has overgrown its normally free lateral edge $(\boldsymbol{d})$. cb, Cerebellum; 8th n, eighth nerve. Scale bar, $100 \mu \mathrm{m}$.

the developing hindbrain, Sef appears to act in cells adjacent to the rhombic lip, thereby restricting activation of the MAP kinase pathway to the rhombic lip itself. Gain-of-function and loss-offunction studies confirm that the proper amount and localization of Sef is necessary for normal development of the auditory nuclei. In adults, Sef is present in astrocytes and may regulate GFAP levels, a role that may also contribute to changes in responses to sound. These results demonstrate that additional investigations of FGF signaling in the auditory brainstem are warranted and may provide a fruitful avenue for elucidating how diverse types of neurons are generated from the rhombic lip.

Sef could normally regulate cochlear nucleus development in two ways: (1) indirectly by restricting signals to the rhombic lip or (2) directly by regulating development of cochlear nucleus precursors from outside of the rhombic lip. In the first model, the primary function of Sef is to limit the effects of FGFs to the rhombic lip. This idea is supported by the fact that Sef is adjacent to the rhombic lip, overlapping with FGF15, and that Sef is known to inhibit response to FGF. Sef mutant cells may therefore become sensitive to FGF signals that would normally be restricted to the rhombic lip. Alternatively, Sef may contribute directly to cochlear nucleus development, because Sef-expressing cells ap- pear to move toward the cochlear nucleus (Fig. 2) and a subset of cochlear nucleus neurons derive from territories ventral to the auditory lip (Willard and Martin, 1986; Farago et al., 2006). In this case, the presence of Sef may change the consequences of FGF signaling in this small subset of cells, perhaps directly affecting their fate in the cochlear nucleus. As suitable markers and tools for fate mapping Sefexpressing cells become available, it will be interesting to determine whether the subpopulation of neurons that comes from outside of the rhombic lip is abnormal in mutant animals and whether Sef is indeed present in precursors for these cells.

The consequences of Sef overexpression in the chick neural tube provide compelling evidence that properly regulated FGF signaling is necessary for normal auditory brainstem development, with more than half of the electroporated embryos displaying obvious defects in NM and/or NA. The variability may be attributable to the fact that Sef is ectopically expressed in a subset of rhombic lip cells, with the number and relative rostrocaudal location of these cells changing between embryos. Attenutation of FGF signaling in other regions of the nervous system simultaneously decreases proliferation and increases cell death (Storm et al., 2006). Thus, the simplest explanation for the observed effects is that there are fewer cochlear nucleus precursors because of a combination of less proliferation and more cell death. This would also fit with the fact that the consequences of Sef overexpression resemble what happens when portions of the rhombic lip are surgically removed (Harkmark, 1954).

An unresolved question is why the brainstem is not more severely affected in Sef ${ }^{\text {KST223 }}$ mutant mice. Because the KST223 strain was generated by gene trap mutagenesis, Sef ${ }^{K S T 223}$ homozygotes produce small amounts of wild-type Sef transcript, raising the possibility that feedback-induced regulation of Sef can still occur. However, our experiments show that even moderate decreases in Seflevels cause strong phenotypes, as demonstrated by the fact that some heterozygotes have abnormal ABRs and severe cochlear nucleus defects (Figs. 5, 6). Similarly, partial knockdown of Sef by small interfering RNA in cell lines has dramatic effects on cell migration and invasion in response to FGF (Darby et al., 2006). Like the Sef ${ }^{K S T 223}$ mice, the conventional Sef knock-out mice are viable with no obvious defects (Lin et al., 2005). Thus, we believe that the defects observed in the KST223 strain reflect a strong loss of Sef function.

The lack of obvious defects in gene trap and conventional Sef knock-out mice may instead reflect the role of Sef as a feedbackinduced antagonist in a pathway that contains multiple regulatory proteins. Deletion of a ligand, receptor, or necessary effector protein generally blocks pathway activation and therefore causes strong mutant phenotypes. In contrast, Sef and other regulatory proteins are not absolutely required for FGF signaling but instead 
affect the degree or timing of pathway activation. Indeed, loss of Spry1 or Spry2 cause mild phenotypes (Basson et al., 2005; Shim et al., 2005; Taketomi et al., 2005). Spry2 overlaps Sef in the brainstem (L. V. Goodrich, unpublished observation) and could suppress the effects of Sef mutations here as occurs in the early embryo (Lin et al., 2005). Thus, depending on how robustly different antagonists or target genes are induced in $S e f^{K S T 223}$ individuals, FGF signaling may be variably affected, leading, in some cases, to severe defects in cochlear nucleus development and, in other cases, to subtle changes in cochlear nucleus function, and causing, at other times, no obvious defects.

The cochlear nucleus defects observed when Sef levels are increased or decreased are consistent with previous reports that FGFs and Sef can affect cell proliferation, migration, or cell death, even within a single cell population. In vitro, Sef overexpression induces apoptosis, whereas knockdown of Sef promotes cell migration in response to FGF ligand, but in vivo roles for Sef have not yet been described (Darby et al., 2006; Kovalenko et al., 2006). FGF2 independently affects both the proliferation and migration of cochlear nucleus precursors in vitro (Zhou et al., 1996; Hossain et al., 2006), whereas FGF8 contributes to tissue morphogenesis, proliferation, and survival in the hindbrain but is surprisingly dispensible for specific cell fate decisions here (Foucher et al., 2006). Similarly, there are no obvious changes in gene expression in Sef mutant embryos, and the effects of Sef overexpression could be explained either by decreased proliferation and/or increased cell death. Conversely, the cochlear nucleus defects observed in Sef ${ }^{\text {KST223 }}$ mutant mice could be caused by a combination of increased proliferation and changes in migration. We therefore favor the idea that in Sef ${ }^{K S T 223}$ mutants, FGF signaling induces a transient upregulation of cell proliferation in the Sef-expression domain, leading, in most cases, to changes that are obvious physiologically but not anatomically. Subtle changes in the proportions of specific cell types or the ability of neurons to integrate properly into auditory circuits would be difficult to detect without highly specific markers. However, even mild changes in organization could lead to detectable changes in auditory function. Because of the partial penetrance of the phenotype, more sensitive methods are necessary to determine whether proliferation, survival, and/or migration are altered in the Sef mutant hindbrain.

We have focused on the developmental functions of Sef, but it is possible that aspects of the hearing impairment reflect an adult function for the FGF pathway. In the adult brain, Sef is present in astrocytes of the cochlear nucleus but not in other regions of the central auditory system (L. V. Goodrich, unpublished observation). This pattern of expression is consistent with the fact that the FGF pathway is active in astrocytes (Luo et al., 1995; Riedel et al., 1995; Smith et al., 2002). Astrocytes are vital components of the synapse and could affect synaptic transmission directly or serve a traditional trophic role in traumatic situations (Newman, 2003). For example, FGF1 and FGF2 have been postulated to play a protective role in response to acoustic overstimulation in the cochlear nucleus (Luo et al., 1995; Riedel et al., 1995; Smith et al., 2002). If FGF signaling serves a protective function, the Sef mutant phenotype might vary depending on factors such as noise exposure and auditory experience.

The auditory phenotype of Sef mutant mice appears to originate in the CNS and may represent a novel model for central auditory processing defects. Most mutants described in the literature are profoundly deaf, with significantly elevated ABR thresholds attributable to defects in the generation, function, or survival of hair cells (Steel et al., 2002). In contrast, mutations that affect central auditory processing are uncommonly reported, and studies of the perceptual consequences in other mouse strains with 


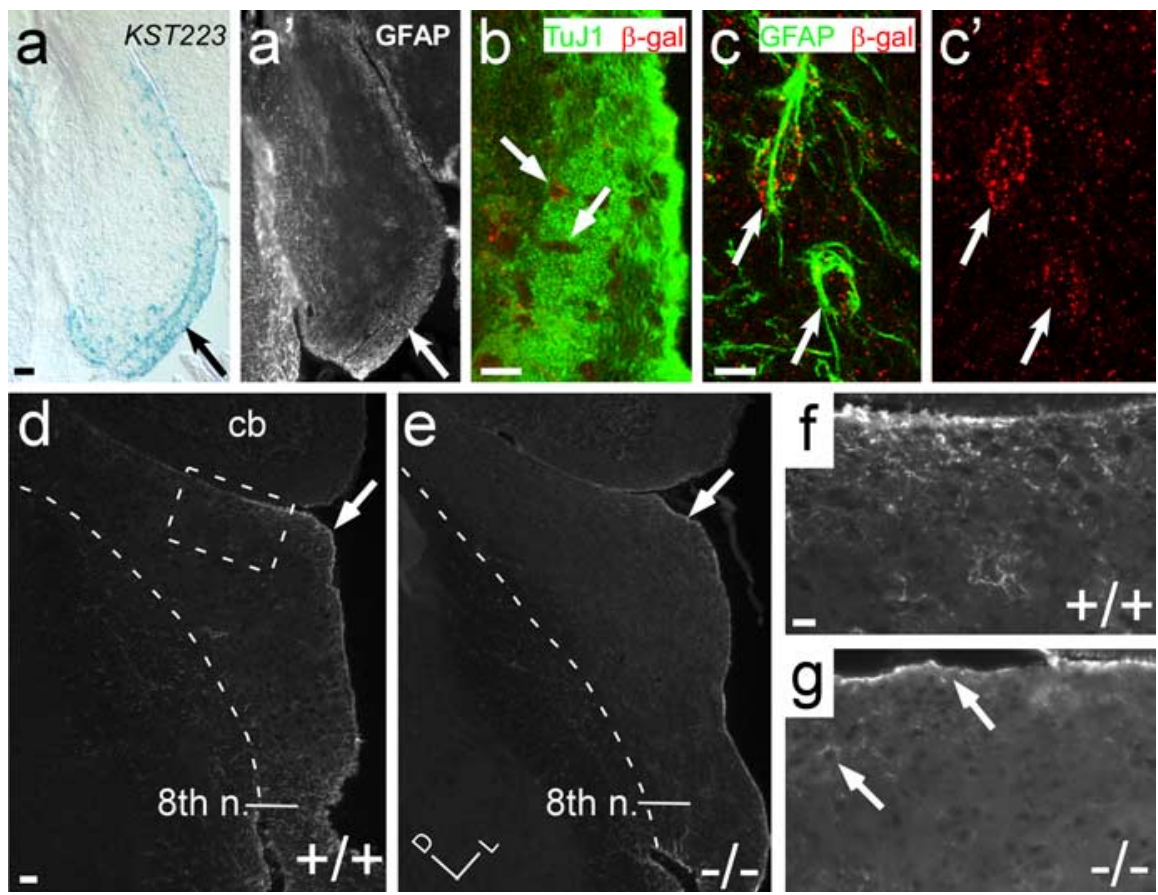

Figure 7. Decreased GFAP levels in cochlear nucleus astrocytes in strongly affected Sef ${ }^{\alpha S T 223}$ mutants. $\boldsymbol{a}, \boldsymbol{a}^{\prime}$, A coronal section through DCN from a P14 Sef ${ }^{\text {KST223 }}$ heterozygote double labeled for $\beta$-galactosidase activity $(\boldsymbol{a})$ and antibodies to GFAP ( $\left.\boldsymbol{a}^{\prime}\right)$. Sef- $\beta$-geo and GFAP have similar patterns of distribution, with intense expression in the molecular layer (arrows). $\boldsymbol{b}$, Double labeling of a P17 Sef ${ }^{\text {KST223 }}$ heterozygote with antibodies to TuJ1 (green) and $\beta$-galactosidase ( $\beta$-gal; red) shows production of Sef- $\beta$-geo in small non-neuronal cells (arrows) in the molecular layer. $\boldsymbol{c}, \boldsymbol{c}^{\prime}$, Merged ( $\boldsymbol{c}$ ) and single channel ( $\boldsymbol{c}^{\prime}$ ) Z series projections of two astrocytes (arrows). Sef- $\beta$-geo (red) is present in a puncate pattern within the cytoplasm of GFAP-positive astrocytes (green). $\beta$-gal, $\beta$-Galactosidase. $\boldsymbol{d}$-g, Anti-GFAP antibody labeling of coronal sections from a wild type $(\boldsymbol{d}, \boldsymbol{f})$ and a Sef $f^{\alpha T 223}$ homozygote $(\boldsymbol{e}, \boldsymbol{g})$ through AVCN at the entry of the eighth nerve (8th n.). Dorsal (D) is up and to the left; lateral (L) is up and to the right. The dashed line indicates the boundary between the cochlear nucleus and the rest of the brainstem. The sections were processed and analyzed in parallel, using the same settings for image capture. High-power views of the granule cell layer are shown in $\boldsymbol{f}$ and $\boldsymbol{g}$ and correspond to the region boxed in $\boldsymbol{d}$. In normal animals, GFAP staining is prominent in the microneuronal shell (arrows) and in the eighth nerve. Although GFAP continues to be expressed at high levels on the surface of the cochlear nucleus and in scattered cells ( $\boldsymbol{g}$, arrows), very little expression is seen in the microneuronal shell of a Sef ${ }^{K S T 223}$ homozygote that had significantly elevated $A B R$ thresholds in both ears ( $55 \mathrm{~dB}$ on the right and $80 \mathrm{~dB}$ on the left for wave iii). cb, Cerebellum. Scale bars: $\boldsymbol{a}, \boldsymbol{b}$, d, $40 \mu \mathrm{m} ; \boldsymbol{c}, 5 \mu \mathrm{m} ; \boldsymbol{f}, 10 \mu \mathrm{m}$.

cochlear nucleus defects have not been performed (Martin, 1981; Liu et al., 2000). However, similar defects in the ABR waveforms recorded from human patients are thought to originate in the auditory brainstem. Children suffering from the lysosomal storage disorder Gaucher disease have perfectly normal hair cell function and hence normal early waves in their ABRs (Lacey and Terplan, 1984; Kaga et al., 1998). However, extensive gliosis and neuronal degeneration in the brainstem causes abnormalities or complete loss of the later waves, comparable to what is seen in Sef ${ }^{K S T 223}$ mutant mice. In addition, people with central auditory processing disorder (CAPD) cannot properly discriminate or localize sound, although their hearing sensitivity is normal. ABR waveforms from CAPD patients have smaller and broader peaks (Gopal and Kowalski, 1999). Nothing is known about the cellular causes of this or other central auditory defects in the human population. Future studies of the auditory processing defects observed in $\mathrm{Sef}^{\text {KST223 }}$ mutant mice may provide useful insights into these important medical questions.

\section{References}

Basson MA, Akbulut S, Watson-Johnson J, Simon R, Carroll TJ, Shakya R, Gross I, Martin GR, Lufkin T, McMahon AP, Wilson PD, Costantini FD, Mason IJ, Licht JD (2005) Sproutyl is a critical regulator of GDNF/RETmediated kidney induction. Dev Cell 8:229-239.
Cramer KS, Fraser SE, Rubel EW (2000) Embryonic origins of auditory brainstem nuclei in the chick hindbrain. Dev Biol 224:138-151.

Cramer KS, Bermingham-McDonogh O, Krull CE, Rubel EW (2004) EphA4 signaling promotes axon segregation in the developing auditory system. Dev Biol 269:26-35.

Cramer KS, Cerretti DP, Siddiqui SA (2006) EphB2 regulates axonal growth at the midline in the developing auditory brainstem. Dev Biol 295:76-89.

Darby S, Sahadevan K, Khan MM, Robson CN Leung HY, Gnanapragasam VJ (2006) Loss of Sef (similar expression to FGF) expression is associated with high grade and metastatic prostate cancer. Oncogene 25:4122-4127.

Dono R (2003) Fibroblast growth factors as regulators of central nervous system development and function. Am J Physiol Regul Integr Comp Physiol 284:R867-R881.

Farago AF, Awatramani RB, Dymecki SM (2006) Assembly of the brainstem cochlear nuclear complex is revealed by intersectional and subtractive genetic fate maps. Neuron 50:205-218.

Foucher I, Mione M, Simeone A, Acampora D, Bally-Cuif L, Houart C (2006) Differentiation of cerebellar cell identities in absence of Fgf signalling in zebrafish Otx morphants. Development 133:1891-1900.

Furthauer M, Lin W, Ang SL, Thisse B, Thisse C (2002) Sef is a feedback-induced antagonist of Ras/MAPK-mediated FGF signalling. Nat Cell Biol 4:170-174.

Gopal KV, Kowalski J (1999) Slope analysis of Auditory Brainstem Responses in children at risk of central auditory processing disorders. Scand Audiol 28:85-90.

Gray PA, Fu H, Luo P, Zhao Q, Yu J, Ferrari A Tenzen T, Yuk DI, Tsung EF, Cai Z, Alberta JA, Cheng LP, Liu Y, Stenman JM, Valerius MT, Billings N, Kim HA, Greenberg ME, McMahon AP, Rowitch DH, et al. (2004) Mouse brain organization revealed through direct genome-scale TF expression analysis. Science 306:2255-2257.

Harduf H, Halperin E, Reshef R, Ron D (2005) Sef is synexpressed with FGFs during chick embryogenesis and its expression is differentially regulated by FGFs in the developing limb. Dev Dyn 233:301-312.

Harkmark W (1954) Cell migrations from the rhombic lip to the inferior olive, the nucleus raphe and the pons. A morphological and experimental investigation of chick embryos. J Comp Neurol 100:115-209.

Hossain WA, D'Sa C, Morest DK (2006) Site-specific interactions of neurotrophin-3 and fibroblast growth factor (FGF2) in the embryonic development of the mouse cochlear nucleus. J Neurobiol 66:897-915.

Kaga K, Shinoda Y, Suzuki JI (1997) Origin of auditory brainstem responses in cats: whole brainstem mapping, and a lesion and HRP study of the inferior colliculus. Acta Otolaryngol 117:197-201.

Kaga K, Ono M, Yakumaru K, Owada M, Mizutani T (1998) Brainstem pathology of infantile Gaucher's disease with only wave I and II of auditory brainstem response. J Laryngol Otol 112:1069-1073.

Kataoka K, Fujiwara KT, Noda M, Nishizawa M (1994) MafB, a new Maf family transcription activator that can associate with Maf and Fos but not with Jun. Mol Cell Biol 14:7581-7591.

Kovalenko D, Yang X, Nadeau RJ, Harkins LK, Friesel R (2003) Sef inhibit fibroblast growth factor signaling by inhibiting FGFR1 tyrosine phosphorylation and subsequent ERK activation. J Biol Chem 278:14087-14091.

Kovalenko D, Yang X, Chen PY, Nadeau RJ, Zubanova O, Pigeon K, Friesel R (2006) A role for extracellular and transmembrane domains of Sef in Sef-mediated inhibition of FGF signaling. Cell Signal 18:1958-1966. 
Krull CE (2004) A primer on using in ovo electroporation to analyze gene function. Dev Dyn 229:433-439.

Lacey DJ, Terplan K (1984) Correlating auditory evoked and brainstem histologic abnormalities in infantile Gaucher's disease. Neurology 34:539-541.

Landsberg RL, Awatramani RB, Hunter NL, Farago AF, DiPietrantonio HJ, Rodriguez CI, Dymecki SM (2005) Hindbrain rhombic lip is comprised of discrete progenitor cell populations allocated by Pax6. Neuron 48:933-947.

Leighton PA, Mitchell KJ, Goodrich LV, Lu X, Pinson K, Scherz P, Skarnes WC, Tessier-Lavigne M (2001) Defining brain wiring patterns and mechanisms through gene trapping in mice. Nature 410:174-179.

Liberman MC, Gao J, He DZ, Wu X, Jia S, Zuo J (2002) Prestin is required for electromotility of the outer hair cell and for the cochlear amplifier. Nature 419:300-304.

Lin W, Furthauer M, Thisse B, Thisse C, Jing N, Ang SL (2002) Cloning of the mouse Sef gene and comparative analysis of its expression with Fgf8 and Spry2 during embryogenesis. Mech Dev 113:163-168.

Lin W, Jing N, Basson MA, Dierich A, Licht J, Ang SL (2005) Synergistic activity of Sef and Sprouty proteins in regulating the expression of Gbx2 in the mid-hindbrain region. Genesis 41:110-115.

Liu M, Pereira FA, Price SD, Chu MJ, Shope C, Himes D, Eatock RA, Brownell WE, Lysakowski A, Tsai MJ (2000) Essential role of BETA2/NeuroD1 in development of the vestibular and auditory systems. Genes Dev 14:2839-2854.

Luo L, Moore JK, Baird A, Ryan AF (1995) Expression of acidic FGF mRNA in rat auditory brainstem during postnatal maturation. Brain Res Dev Brain Res 86:24-34.

Machold R, Fishell G (2005) Math1 is expressed in temporally discrete pools of cerebellar rhombic-lip neural progenitors. Neuron 48:17-24.

Martin MR (1981) Morphology of the cochlear nucleus of the normal and reeler mutant mouse. J Comp Neurol 197:141-152.

Melcher JR, Kiang NY (1996) Generators of the brainstem auditory evoked potential in cat. III: Identified cell populations. Hear Res 93:52-71.

Melcher JR, Knudson IM, Fullerton BC, Guinan Jr JJ, Norris BE, Kiang NY (1996a) Generators of the brainstem auditory evoked potential in cat. I. An experimental approach to their identification. Hear Res 93:1-27.

Melcher JR, Guinan Jr JJ, Knudson IM, Kiang NY (1996b) Generators of the brainstem auditory evoked potential in cat. II. Correlating lesion sites with waveform changes. Hear Res 93:28-51.

Mitchell KJ, Pinson KI, Kelly OG, Brennan J, Zupicich J, Scherz P, Leighton PA, Goodrich LV, Lu X, Avery BJ, Tate P, Dill K, Pangilinan E, Wakenight P, Tessier-Lavigne M, Skarnes WC (2001) Functional analysis of secreted and transmembrane proteins critical to mouse development. Nat Genet 28:241-249.

Newman EA (2003) New roles for astrocytes: regulation of synaptic transmission. Trends Neurosci 26:536-542.

Peters K, Ornitz D, Werner S, Williams L (1993) Unique expression pattern of the FGF receptor 3 gene during mouse organogenesis. Dev Biol 155:423-430.

Peters KG, Werner S, Chen G, Williams LT (1992) Two FGF receptor genes are differentially expressed in epithelial and mesenchymal tissues during limb formation and organogenesis in the mouse. Development 114:233-243.

Pierce ET (1967) Histogenesis of the dorsal and ventral cochlear nuclei in the mouse. An autoradiographic study. J Comp Neurol 131:27-54.

Preger E, Ziv I, Shabtay A, Sher I, Tsang M, Dawid IB, Altuvia Y, Ron D (2004) Alternative splicing generates an isoform of the human Sef gene with altered subcellular localization and specificity. Proc Natl Acad Sci USA 101:1229-1234.

Pringle NP, Yu WP, Howell M, Colvin JS, Ornitz DM, Richardson WD (2003) Fgfr3 expression by astrocytes and their precursors: evidence that astrocytes and oligodendrocytes originate in distinct neuroepithelial domains. Development 130:93-102.

Riedel B, Friauf E, Grothe C, Unsicker K (1995) Fibroblast growth factor-2like immunoreactivity in auditory brainstem nuclei of the developing and adult rat: correlation with onset and loss of hearing. J Comp Neurol 354:353-360.

Rodriguez CI, Dymecki SM (2000) Origin of the precerebellar system. Neuron 27:475-486.

Ryugo DK, Parks TN (2003) Primary innervation of the avian and mammalian cochlear nucleus. Brain Res Bull 60:435-456.

Sato T, Nakamura H (2004) The Fgf8 signal causes cerebellar differentiation by activating the Ras-ERK signaling pathway. Development 131:4275-4285.

Shim K, Minowada G, Coling DE, Martin GR (2005) Sprouty2, a mouse deafness gene, regulates cell fate decisions in the auditory sensory epithelium by antagonizing FGF signaling. Dev Cell 8:553-564.

Smith L, Gross J, Morest DK (2002) Fibroblast growth factors (FGFs) in the cochlear nucleus of the adult mouse following acoustic overstimulation. Hear Res 169:1-12.

Steel K, Erven A, Kiernan A (2002) Mice as models for human hereditary deafness. In: Genetics and auditory disorders (Keats BJB, Popper AN, Fay RR, eds), pp 247-296. New York: Springer.

Storm EE, Garel S, Borello U, Hebert JM, Martinez S, McConnell SK, Martin GR, Rubenstein JL (2006) Dose-dependent functions of Fgf8 in regulating telencephalic patterning centers. Development 133:1831-1844.

Taketomi T, Yoshiga D, Taniguchi K, Kobayashi T, Nonami A, Kato R, Sasaki M, Sasaki A, Ishibashi H, Moriyama M, Nakamura KI, Nishimura J, Yoshimura A (2005) Loss of mammalian Sprouty2 leads to enteric neuronal hyperplasia and esophageal achalasia. Nat Neurosci 8:855-857.

Timmer JR, Wang C, Niswander L (2002) BMP signaling patterns the dorsal and intermediate neural tube via regulation of homeobox and helix-loophelix transcription factors. Development 129:2459-2472.

Torii S, Kusakabe M, Yamamoto T, Maekawa M, Nishida E (2004) Sef is a spatial regulator for Ras/MAP kinase signaling. Dev Cell 7:33-44.

Tsang M, Dawid IB (2004) Promotion and attenuation of FGF signaling through the Ras-MAPK pathway. Sci STKE 2004:pe17.

Tsang M, Friesel R, Kudoh T, Dawid IB (2002) Identification of Sef, a novel modulator of FGF signalling. Nat Cell Biol 4:165-169.

Wang VY, Rose MF, Zoghbi HY (2005) Math1 expression redefines the rhombic lip derivatives and reveals novel lineages within the brainstem and cerebellum. Neuron 48:31-43.

Willard FH, Martin GF (1986) The development and migration of large multipolar neurons into the cochlear nucleus of the North American opossum. J Comp Neurol 248:119-132.

Wingate RJ (2001) The rhombic lip and early cerebellar development. Curr Opin Neurobiol 11:82-88.

Wright TJ, Ladher R, McWhirter J, Murre C, Schoenwolf GC, Mansour SL (2004) Mouse FGF15 is the ortholog of human and chick FGF19, but is not uniquely required for otic induction. Dev Biol 269:264-275.

Yamaguchi TP, Conlon RA, Rossant J (1992) Expression of the fibroblast growth factor receptor FGFR-1/flg during gastrulation and segmentation in the mouse embryo. Dev Biol 152:75-88.

Yang RB, Ng CK, Wasserman SM, Komuves LG, Gerritsen ME, Topper JN (2003) A novel interleukin-17 receptor-like protein identified in human umbilical vein endothelial cells antagonizes basic fibroblast growth factor-induced signaling. J Biol Chem 278:33232-33238.

Zaaroor M, Starr A (1991) Auditory brain-stem evoked potentials in cat after kainic acid induced neuronal loss. II. Cochlear nucleus. Electroencephalogr Clin Neurophysiol 80:436-445.

Zhou X, Hossain WA, Rutledge A, Baier C, Morest DK (1996) Basic fibroblast growth factor (FGF-2) affects development of acoustico-vestibular neurons in the chick embryo brain in vitro. Hear Res 101:187-207. 